\title{
GESTIÓN DE LAS EMPRESAS FAMILIARES: RETOS Y OPORTUNIDADES
}

\author{
MANAGEMENT OF FAMILY BUSINESS: \\ CHALLENGES AND OPPORTUNITIES
}

\author{
Harold Yusit Gamero Maldonado \\ Universidad Católica San Pablo, Arequipa, Perú
}

\section{Resumen:}

Las empresas familiares tienen sus origines, prácticamente, en concomitancia con los orígenes de la familia misma y las sociedades. Estas organizaciones han avanzado de forma estable y hoy en día se consolidan como la mayor fuente de ingresos y puestos de trabajo en los países más importantes del mundo globalizado. Lamentablemente, por su naturaleza, estas empresas también enfrentan una serie de retos que sus contrapartes no familiares, no experimentan; haciendo que éstas empresas tengan tiempos de vida muchos menores. Por tal razón, es menester atender a los requerimientos particulares de este tipo de empresas para poder aumentar las probabilidades -si no es garantizar- la sucesión de dichos organismos a través de futuras generaciones, idealmente, bajo la dirección de personas ajenas a la familia para evitar que los conflictos consanguíneos se incrusten en las operaciones de la empresa. Finalmente se encuentra que, dentro de las herramientas de gestión que dispone el fundador, la más útil de todas es la del protocolo familiar.

Palabras clave: Empresa familiar, gerencia y administración de empresas familiares, sucesión generacional, protocolo familiar.

\section{Summary:}

Family business have their origins practically in conjunction with the origins of family and society. These organizations have progressed firmly and today are consolidated as the largest source of revenues and job creations in the most important countries in the 
globalized world. Unfortunately, because their nature, these companies also face various challenges than their non-family counterparts; making the lifetime expectations of these ones shorter. For these reasons, it is mandatory to respond to their particular requirements, in order to increase the odds -if it is not guarantee- the succession of these bodies through future generations; ideally under the management of an outsider, so the conflicts between consanguineous actors can be prevented, and so, the conflicts inside the business. Finally, it has been found that the most useful management tool, is the family protocol.

Keywords: Family business, management and administration of family businesses, generational succession, family protocol.

\section{Introducción}

El término "empresa” tiene sus orígenes etimológicos en los vocablos latinos in, "en” y prehendere, "acometer"; que en conjunto se podrían entender como la decisión de comenzar a hacer algo o de actuar. En un contexto más reciente y especializado, la empresa se define como "una unidad económica que desarrolla actividades productivas, a través de la combinación de factores, los cuales permiten generar bienes o servicios, sobre la base de alcanzar un beneficio privado o colectivo" (Hernández, 2011, p. 66). En base a estas definiciones podríamos considerar a la misma familia como una empresa, y en efecto lo es; sin embargo, en la evolución del lenguaje, éste término ha ido aterrizando en las parcelas del vocabulario empresarial, por lo que hoy en día, hablar de empresas familiares ya no es redundar, sino precisar.

En los últimos años, las empresas familiares han avanzado hasta ser partícipes de un gran porcentaje del total de empresas en el mundo, así como de contribuir sustancialmente al desarrollo de los países económicamente más avanzados (Upton \& Petty, 2000; Amat, 2001; Chua et al., 2003; Duréndez \& García, 2005). Aunque por lo general son pequeñas, en conjunto las empresas familiares representan una fracción importante de las empresas más poderosas de la economía global y contribuyen considerablemente a la formación de empleo (Aronoff et al., 1996; Neuberg \& Lank, 1998). En el Perú, las empresas familiares también son mayoría, puesto que estudios recientes han encontrado que éste tipo de empresas representan el $80 \%$ del total de empresas peruanas, las cuales generan el 6o\% del empleo nacional (Jaramillo, 2014).

Sin embargo, las empresas familiares presentan un tiempo de vida menor al que sostienen sus contrapartes no familiares: en un estudio realizado por el Instituto de Empresa de España, solo el 30 por ciento de las empresas familiares en el Perú supera la expectativa de vida de 24 años, que es en promedio el periodo durante el cual el fundador tiene el control de la empresa. Es así como otras investigaciones han encontrado que en promedio sólo el 30\% de esas empresas pasa a manos de la segunda generación y sólo un cinco por ciento llegan a la tercera (Jaramillo, 2014). La situación también es similar en otros países más desarrollados; por ejemplo, en Canadá, investigadores han 
encontrado que también un treinta por ciento de empresas familiares sobrevive a la generación que las funda; y que muchas de las sucesiones intergeneracionales fracasa poco después que la segunda generación toma el control (Miller, Steier \& Le Breton-Miller, 2003).

Estos bajos índices de sucesión se consolidan como las trabas más grandes para el desarrollo y crecimiento de las empresas familiares o FOBs por sus siglas en inglés (Family Owned Businesses) (Carlock \& Ward, 2001), por lo que el presente artículo se desarrolla bajo esta premisa y, a la luz de investigaciones previas, revisa las causas más comunes y plantea recomendaciones, a fin de apoyar a las familias emprendedoras en la correcta gestión de sus organizaciones. Para tal fin, en el primer apartado se encuentra una revisión teórica de lo que son las empresas familiares: su definición y características; luego se estudian los retos que tienen las mismas; y finalmente, se dan las recomendaciones y pautas que la ciencia y la experiencia empírica, ha podido descubrir para asegurar -o al menos maximizar- la sucesión de este tipo de empresas.

\section{Definición y características de las empre- sas familiares}

Algunas investigaciones (Wortman, 1994, 1995; Smyrnios \& Romano, 1994; Smyrnios et al., 1997; Stoy Hayward \& London Business School, 1989, 1990) han encontrado que existen más de 20 definiciones de empresas familiares (únicamente en la lengua inglesa). Una de las primeras definiciones sobre este tipo de empresas fue realizada por Donnelley en 1964, en la cual se plantean siete requisitos a cumplir para que una empresa pueda ser denominada como familiar. Entre estos siete indicadores se mencionan: la priorización de familiares para la sucesión de la empresa, la presencia de familiares en la junta directiva, el reflejo de los valores y reputación familiar en el negocio, los sentimientos de obligación de los miembros de la familia a mantener las acciones de la empresa, entre otros. Sin embargo, esta definición extensa e imprecisa dificultó su utilización para investigaciones empíricas por lo que no se utilizó para estudios posteriores (Zachary, 2011).

Dada ésta falta de claridad en las definiciones, un grupo de investigadores de la universidad de Monash en Australia, encontraron que las empresas familiares son aquellas que cumplen alguna de las siguientes tres características (Romano, Tanewski \& Smyrnios, 20oo):

a) El 50 por ciento o más del capital de la empresa, pertenece a una familia o varios miembros de varias familias;

b) una familia es, efectivamente, la que controla el negocio; y

c) una fracción importante de la dirección o gestión de la empresa recae en una misma familia.

Otra aproximación, más estricta es la que plantea Sharma et al. (2007, en Duréndez \& García, 2005, p. 242), la cual indica que las empresas familiares son aquellas que cumplen, a la vez, todas las siguientes características:

a) "Propiedad y control de la empresa en la misma familia; 
b) Influencia de la familia en la toma de decisiones; $y$

c) Propósito de transmitir la empresa a la siguiente generación."

Por consiguiente, se puede concluir que las empresas familiares, son aquellos negocios formados y gobernados por un grupo dominante de parientes, que en sentido estricto serían los miembros de una misma familia (o una pequeña cantidad de ellas), con la finalidad de continuar con la visión de la empresa a través de las generaciones de la o las familias (Chua, Chrisman \& Sharma, 1999, en Navarro de Granadillo, 2008). Es así, que la característica principal de toda empresa familiar es el nivel de participación que tiene una familia, en la propiedad y/o dirección de la empresa (Brenes, Madrigal \& Molina, 2006).

\section{Retos en las empresas familiares}

Diversos estudios concuerdan en que el reto más grande que enfrentan éste tipo de empresas se generan debido a su propia naturaleza: la estrecha relación entre familia y empresa (Donnelley, 1964; Zachary, 2011; Romano, Tanewski y Smyrnios, 200o). Dentro de esta interacción, uno de los procesos cruciales que suele verse comprometido por esta situación es la toma de decisiones en la dirección de la empresa. Según Beckhard y Gibb (1983) las decisiones que se toman en las empresas familiares por lo general están supeditadas a dos tipos de factores: los propios de la dinámica comercial/organizacional y otros factores familiares. Los tres principales factores organizacionales son: el ambiente general de la organización, el grado de desarrollo de la firma y la cultura (normas y tradiciones) de la propia organización; mientras que los factores que afectan desde el aspecto familiar son: la cultura de la familia, la influencia de la familia del fundador y los valores y motivaciones del mismo fundador.

A raíz de estas circunstancias, las mismas decisiones del fundador pueden verse comprometidas debido a que, en muchos de estos negocios, los fundadores hacen lo posible por que sus descendientes tomen la posta de la empresa, y así garantizar su legado y el control de la familia sobre las decisiones de la empresa (Miller, Steier \& Le Breton-Miller, 2003). Lamentablemente, este tipo de decisiones impide designar objetivamente a la persona más adecuada para seguir con la gestión, lo que contribuye a los bajos índices de sucesión revisados anteriormente. Este comportamiento hace de la sucesión intergeneracional un factor crucial para el éxito de las empresas familiares.

Diversas investigaciones sugieren que son varios los factores de por quéla sucesión de una empresa familiar fracasa, las cuales incluyen la incompetencia o poca preparación de los sucesores, los conflictos familiares internos o la ausencia o poca preparación de un plan de traspaso generacional (Handler, 1994; Miller, Steier \& Le Breton-Miller, 2003). Sobre esta última, se puede mencionar que existen altas probabilidades de que los hijos, quienes son los sucesores favoritos de los fundadores, tengan aptitudes, capacidades e intereses diferentes a las de su padre, o carezcan de habilidades y conocimientos suficientes para la efectiva conducción y consecución de los objetivos de la empresa.

Adicionalmente, hay que considerar que la brecha entre el antiguo gerente y el 
nuevo, al momento de la transición, suele ser muy grande, presentándose normalmente una diferencia entre 25 y 35 años (Handler, 1994). Esta brecha también suele significar que el sucesor no tiene la madurez suficiente para manejar todas las responsabilidades que la empresa demanda, pudiendo devenir en tres tipos diferentes de actitudes: sobredependencia hacia el padre y conservadurismo, rebeldía y predisposición excesiva al cambio, o confusión e inseguridad. Si bien en cierto grado, puede ser beneficioso para la organización que los líderes que tomen la dirección tengan diferentes personalidades, es indispensable que todos los sucesores al menos mantengan los mismos conocimientos y habilidades para generar estratégicas apropiadas, para mantener o mejorar la cultura organizacional, y para gobernar correcta y eficientemente.

Con relación a los conflictos internos que pueden experimentar las empresas familiares, a menudo se encuentra que, si bien este tipo de empresas busca alcanzar un equilibrio entre la familia y la empresa, usualmente esta situación es difícil de alcanzar para muchas de estas empresas (Brenes \& Madrigal, 2003). Al tener una estrecha relación entre ambas, normalmente, cuando las necesidades y el bienestar de la familia toma importancia, ésta suele darse en detrimento del futuro crecimiento de la empresa; por el contrario, cuando se le da prioridad a la compañía, usualmente se perjudica la comunicación, la armonía y satisfacción de las necesidad y problemas familiares (Carlock and Ward, 2001). La efectiva elección del sucesor adecuado para la empresa, suele influir también en el balance adecuado entre familia y empresa (Romero, 2001).
KPMG (2011), una de las firmas de asesoría empresarial más importarte del mundo, menciona que los principales retos que enfrentan las empresas familiares, se originan tras la expansión del árbol genealógico de la familia, generando que la cantidad de personas involucradas en la empresa sea mayor; y, a su vez, esta situación, puede traer consigo los siguientes conflictos: a) conflicto de metas y objetivos, puesto que los miembros de la familia pueden tener metas y valores muy diferentes; b) conflictos personales, debido a la particularidad de cada individuo. Lo que puede generar malos entendidos y problemas entre los miembros trabajadores o entre generaciones, los cuales podrían destruir la armonía laboral o incluso la empresa; c) distintas expectativas de las familias y sus integrantes, sobre todo en cuanto a lo que esperan de la empresa: salario, cargos, actividades, tareas, etc.; d) dificultades en la selección de trabajadores idóneos para los diferentes puestos dentro de empresa (lo que supone en muchos casos, elegir entre varios miembros de la familia); e) la repartición de utilidades, las cuales suelen ser el mayor reto para las empresas familiares; y f) casi siempre los fundadores no poseen los conocimientos suficientes, ni le dan la importancia debida a los planes estratégicos, de sucesión o financieros, por lo que se genera una renuencia a planificar.

Según el Instituto para Empresas Familiares (IFB por sus siglas en ingles), la resistencia a la planificación intergeneracional se genera en tres ejes básicos de la organización: en el propietario, en la familia y entre los trabajadores y los factores den entorno. En la Tabla 1 se explican cada una de ellas. 
Otro de los aspectos cruciales a los que se enfrentan este tipo de organizaciones son los relacionados al manejo financiero. En el caso particular del Perú encontramos que en el país existe un gran índice de informalidad que ataca principalmente al sector de micro y pequeñas empresas, las cuales están constituidas en su mayoría por familias emprendedoras (Chacaltana, Bermedo y Gómez, 2008).

Según la Comisión de la Producción del Congreso Peruano, durante estos últimos 4 años, el 40\% de los nuevos emprendedores en el Perú desaparecen o se pasan a la informalidad, debido a que no pueden aguantar la presión tributaría durante los primeros años de vida
(Neyra, 2012). Esto quiere decir que las empresas familiares, que en su mayoría inician sus actividades en la base de las categorías empresariales, enfrentan una serie de dificultades como los son los altos impuestos y las fuertes cargas laborales que exige cada trabajador ingresado a planilla. Si bien estas dificultades también las enfrentan todas las demás empresas no familiares -que ingresan a un mercado cada vez más competitivo- las empresas familiares suelen tener retos adicionales en el transcurso de sus operaciones. Una de ellas es la estructura financiera, puesto que las empresas familiares sostienen sobre sus operaciones, no solo las responsabilidades propias de la empresa, sino también las de la familia (Bermejo, 2008).

Tabla 1.

Resistencia a la planificación intergeneracional: Los 3 ejes fundamentales

\begin{tabular}{|c|c|c|}
\hline Propietario & Familia & $\begin{array}{c}\text { Trabajadores y factores del } \\
\text { entorno }\end{array}$ \\
\hline $\begin{array}{l}\text { - } \text { Miedoa la pérdida de notoriedad. } \\
\text { - } \text { Renuencia a dejar el poder y el } \\
\text { - } \text { Pérdida de identidad, debido a que } \\
\text { muchos propietarios tienen una } \\
\text { fuerteidentificaciónsonsuempresa. } \\
\text { - Predisposición a actuar antes } \\
\text { que planificar. } \\
\text { - Incapacidad de elegir entre sus } \\
\text { hijos al sucesor más adecuado. } \\
\text { - Miedoal retiro, ya que sus empre- } \\
\text { sas suelen convertirse en una } \\
\text { parte predominante de sus vidas. } \\
\text { - Celos y rivalidad entre potencia- } \\
\text { les sucesores. }\end{array}$ & 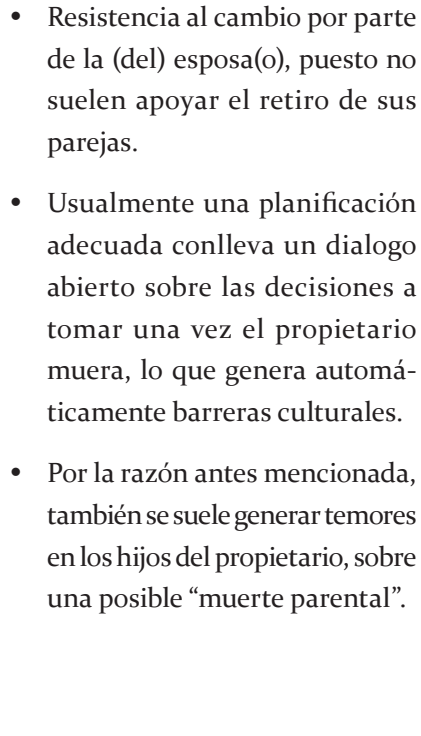 & $\begin{array}{l}\text { - Inseguridad laboral: Para } \\
\text { muchos de los trabajadores } \\
\text { (sobre todo los más antiguos), } \\
\text { su ventaja más grande suele ser } \\
\text { su relación con el propietario, por } \\
\text { lo que un sucesor suele ser visto } \\
\text { como una amenaza. } \\
\text { - Fuera de la empresa, algunos } \\
\text { clientes importantes también } \\
\text { pueden presentar cierta resis- } \\
\text { tencia a confiar en una nueva } \\
\text { gestión. }\end{array}$ \\
\hline
\end{tabular}

Fuente: Adaptado de Institute for Family Business (2008). 
En esta misma línea, se puede encontrar que las empresas familiares prefieren las fuentes de financiamiento internas que las externas. Esto se debe principalmente a que las familias por general tienden a sentir temor a perder el control de la compañía a manos de instituciones financieras o nuevos accionistas, ya que muy a menudo las necesidades y el bienestar de la familia son priorizadas por encima de los requerimientos y crecimiento de la empresa (Carlock \& Ward, 2001). Paradójicamente, los estudios demuestran que una de las causas por la que fracasan las empresas familiares, es la carencia de recursos financieros (en Esparza, García-Pérez \& Duréndez, 2010).

Esta situación se presenta principalmente porque las familias crean una empresa, a la espera de recibir algún tipo de valor como retribución (Chua, Chrisman y Steier, 2003); ya que algunas investigaciones foráneas han encontrado que las familias que deciden fundar y mantener una organización con fines de lucro, tienen como motivación principal una necesidad económica o de sustento vital para la familia; aunque otras también se crean con el objetivo de acumular una mayor riqueza, alcanzar una mejor calidad de vida o, inclusive, satisfacer necesidades individuales o de autorrealización, entre otras (Winter et al., 1998; Zachary, 2011).

\section{Recomendaciones para la sucesión de la empresa familiar:}

\section{El protocolo familiar}

Si bien las empresas familiares tienen muchas dificultades al inicio, las que sí logran pasar la barrera generacional con eficiencia suelen durar muchos más años que sus contrapartes no familiares. Algunos estudios realizados sobre las empresas más antiguas del mundo, se encontró que muchas de estas eran empresas familiares; en una recopilación realizada por O’Hara y Mandel (2004), se encontró que algunas de las empresas más antiguas del mundo son: Kongo Gumi (fundada en Japón, en el año 578); Hoshi (Japón, 718), Château de Goulaine (Francia, año 1000), Barone Ricasoli (Italia, 1141), Barovier \& Toso (Italia, 1295), Hotel Pilgrim Haus (Alemania, 1304), Richard de Bas (Francia, 1326), Torrini Firenze (Italia, 1369), Antinori (Italia, 1285), Camuffo (Italia, 1438).

Para poder generar los niveles de sostenibilidad antes mencionados, se considera que lo más importante en una empresa familiar es la creación de un protocolo familiar, el cual es un instrumento que facilita la regulación de las interacciones de la familia dentro de la misma empresa, a través de acuerdos voluntarios; el objetivo principal de dicho documento es el de garantizar la continuidad de la empresa a través de las subsiguientes generaciones (Hernández, 2011). En su formato más elemental, el protocolo familiar es una herramienta compuesta por elementos económicos, jurídicos, empresariales y hasta volitivos (o emocionales), siendo este último el más relevante (Díaz \& Fuentes, 2013).

Finalmente, sobre éste tema, Antognolli (2012) menciona que el protocolo familiar debe tener al menos los siguientes elementos: a) debe definir cuándo y en qué circunstancias los hijos podrán trabajar 
en la empresa; b) desarrolla los valores y creencias que se desean mantener en la empresa; c) debe precisar la visión del grupo sobre el futuro de la empresa; d) define el tipo de empresa familiar que se quiere consolidar (tamaño, tipo de negocios, etc.); e) detalla el o los métodos de evaluación del desempeño de los familiares que se va a realizar en la empresa familiar; f) define un marco de evaluación que contemple los resultados laborales alcanzados; g) menciona cómo será la promoción, cómo se accederá a la presidencia o gerencia, si podrá acceder un no familiar, etc.; h) menciona los criterios para la fijación de remuneraciones; i) establece un sistema en términos de competencia laboral que evite romper la armonía en el grupo familiar; j) detalla un método de resolución de conflictos; k) describe los derechos y atribuciones de cada uno de los miembros de la familia; 1) precisa cómo se accede a la propiedad, los criterios para la fijación del precio de las acciones y las regulaciones para la venta de acciones; y $\mathrm{m}$ ) define como serán atendidas las necesidades de los familiares, estableciendo la política de dividendos de la empresa en términos de las necesidades que puedan ir surgiendo.

\section{Conclusiones}

Los diversos hallazgos encontrados indican que las empresas familiares juegan un papel importantísimo para el desarrollo económico de la mayoría de países del mundo, generando una gran cantidad de fuentes de ingresos para el estado y para la sociedad, a través de la generación de fuentes de empleo, pagando impuestos e incrementando la producción interna. Lamentablemente, muchos de estos estudios, también indican que este tipo de organizaciones tienes las tasas de sobrevivencia más bajas en el mercado.

Por otro lado se ha observado que para una eficiente transición generacional, es necesario hacer una definición clara y anticipada de los posibles conflictos que se pueden dar en la empresa, sobre a todo en la interacción entre empresa y familia (Brenes \& Madrigal, 2003). Adicionalmente, se ha observado que una de las etapas cruciales en el desarrollo de la organización es la eficiente y objetiva toma de decisiones; proceso para el cual los expertos recomiendan la participación de personas ajenas a la familia (Brenes, Madrigal \& Molina, 2006), esta decisión evita, además, la generación de conflictos entre los miembros de la familia.

Por el otro lado, tanto las investigaciones desarrolladas en el campo, como la experiencia empresarial de varias corporaciones, indican que una de las herramientas más importantes y poderosas para maximizar la sucesión exitosa de la empresa, es el protocolo familiar (Antognolli, 2012; Brenes, Madrigal \& Molina-Navarro, 2006; Romano, Tanewski \& Smyrnios, 200o) dentro de los cuales deben identificar y conocer aspectos como: el tamaño de la empresa, la industria en la cual se desenvuelven, la antigüedad de la firma, el nivel de control familiar, la edad del fundador, objetivos y metas de la organización, entre otros; para poder así potenciarlas y obtener el mayor provecho de sus empresas, ya que en éstas existe un gran potencial. 


\section{Referencias}

Amat, J. M. (2001). "La continuidad de la empresa familiar". (3ra ed.). Barcelona: Ediciones Gestión 2000.

Antognolli, S. (2012). "El protocolo familiar". Artículo electrónico extraído de http://www.degerencia.com/articulo/ el_protocolo_familiar

Aronoff, C. E.; Astrachan, J. H. \& Ward, J. L. (1996). "Family business sourcebook II. Business owner resources”. Georgia: Marietta.

Bermejo, M. (2008). "Hacia la empresa familiar líder: Una visión práctica y optimista”. España: Prentice-Hall

Brenes E, Madrigal K. (2003). Anticipando el conflicto en los negocios familiares. “Revista INCAE”, 13(3), 372-374.

Brenes, E.; Madrigal, K. \& Molina-Navarro, G. (2006). Family Business Structure and Succession: Critical Topics in Latin American Experience. "Journal of Business Research”, 59(1), 372-374.

Carlock, R. \& Ward, J. (2001). "Strategy planning for the family business: parallel planning to unify the family and business". Londres: London' Palgrave.

Chacaltana, J.; Bernedo, J. \& Gómez, M. (2008). Una evaluación del régimen especial para la microempresa en Perú, al cuarto año de vigencia. "Oficina Internacional del Trabajo (OIT)”. Manuscrito no publicado.
Chua, J.; Chrisman, J. \& Steier, L. (2003). Extending the Theoretical Horizons of Family Business Research. "Entrepreneurship Theory \& Practice", 27(4), 331-338.

Díaz, H. \& Fuentes, E. (2013). La comunicación en la empresa familiar, principal elemento en la preservación de la armonía familiar. "Revista Internacional Administración \& Finanzas”, 6(3), 95-113.

Duréndez, A. \& García, D. (2005). Diferencias económico-financieras entre las empresas familiares y las no familiares. "Revista de Contabilidad y Dirección”, 2(1), 241-265.

Esparza, A.; García-Pérez, D. \& Duréndez, G. (2010). Diferencias de gestión financiera entre empresas familiares y no familiares del sector turístico mexicano. "Actualidad Contable FACES", 20(2), 29-48.

Handler, W., 1994. Succession in family business: a review of the research. “Family Business Review”, 7(1), 133-174.

Hernández, B. (2011). “Compendio Bursátil”. Madrid: Ediciones Díaz de Santos.

Institute for Family Business (2008). "Family business management perspectives: Succession”. Londres: Grant Gordon.

Jaramillo, F. (2014). "Presentación del documento de sistematización del Programa de Desarrollo de Sistemas de Gobierno y Gestión de Empresas 
de Propiedad Familiar". Conferencia del Programa de Desarrollo de Sistemas de Gobierno y Gestión de Empresas de Propiedad Familiar, organizada por la Cámara de Comercio de Lima \& Banco Interamericano de Desarrollo, 26 de noviembre, Lima, Perú.

KMPG (2011). "Family business succession: Managing the all-important family component". Canada: KPMG LLP.

Miller, D.; Steier, L. \& Le Breton-Miller, I. (2003). Lost in time: intergenerational succession, change, and failure in family business. "Journal of Business Venturing", 18(1), 513-531.

Navarro de Granadillo, K. (2008). Estado actual de la investigación sobre la gestión del conocimiento en empresas familiares. "Revista de Ciencias Sociales (RCS)". 14(1), 30-45.

Neuberg, F. \& Lank, A.G. (1998). "The family business: Its governance for sustainability". Londres: Macmillan.

Neyra, A. (2012). Ampliación de noticias de RPP Noticias. Emitido el 8 de mayo del 2012. Recuperado el 5 de abril del 2013 de: http://www.larepublica.pe/ tag/emprendedores-pag4

O'Hara, W. T. \& Mandel, P. (2004). "The world's oldest family companies". España: Universidad de León.

Romano, C.; Tanewski, G. \& Smyrnios, K. (200o). Capital structure decision making: a model for family business.
"Journal of Business Venturing", 16(1), 285-310.

Smyrnios, K. \& Romano, C. (1994). “The Price Waterhouse/Commonwealth Bank Family Business Survey: 1994". Syme Departamento de Contabilidad, Universidad de Monash.

Smyrnios, K., Romano, C. \& Tanewski, G.A. (1997). "The Australian \& Private Family Business Survey: 1997". Melbourne: The National Mutual Family Business Research Unit.

Stoy Hayward \& London Business School (1989). "Staying the course: Survival characteristics of the family owned business". London: Author.

Stoy Hayward \& London Business School (1990). "Managing the Family Business in the UK". London: Author.

Upton, N. \& Petty, W. (200o). Venture capital investment and US family business. "Venture Capital", 2(1), 27-39.

Winter, M.; Fitzgerald, M.; Heck, R.; Haynes, G. \& Danes, S. (1998). Revisiting the study of family businesses: methodological challenges, dilemmas, and alternative approaches. "Family Business Review", 11(3), 239-52.

Wortman, M. (1994). Theoretical foundation for family owned business: A conceptual and research based paradigm. "Family Business Review", 8(1), 3-27. 
Wortman, M. (1995). "Critical issues in Zachary, R. (2011). The importance of the family business: An international family system in family business. perspective of practice and research". "Journal of Family Business ManagePonencia de la $40^{t a}$ Conferencia ment", 1(1), 26-36.

Internacional de Pequeños Negocios, 12 junio, Sydney, Australia. 